\title{
On the absolute value of the density fluctuation measured by reflectometry
}

\author{
M. Schubert, ${ }^{1}$ S. Heuraux,${ }^{1}$ E. Gusakov, ${ }^{2}$ A. Popov, ${ }^{2}$ T. Gerbaud,${ }^{3}$ F. da Silva, ${ }^{4}$ \\ ${ }^{1}$ LPMIA UMR 7040, UHP Nancy 1, BP 239, 54506 Vandouvre Cedex, France \\ ${ }^{2}$ Ioffe Institute, Politekhnicheskaya 26, 194021 St.Petersburg, Russia \\ ${ }^{3}$ Association EURATOM/CEA, CEA/DRFC Cadarache, 13108 St Paul-lez-Durance, France \\ ${ }^{4}$ Associação EURATOM/IST, Centro de Fusão Nuclear, 1046-001 Lisboa, Portugal \\ Martin.Schubert@1pmi.uhp-nancy.fr
}

\section{Introduction}

Microwave reflectometry ${ }^{1}$ is one of the few non-intrusive diagnostics that can be applied to measure density profiles and fluctuations in fusion plasmas. Since the temporal resolution is sufficiently high the reflectometry data are an important input for the modelling and understanding of plasma turbulence. It is, however, still under discussion ${ }^{2}$ how the absolute value of the density fluctuation at the cut-off can be deduced from measured phase fluctuations. There are additional contributions of small angle forward scattering and of resonant Bragg backscattering, both located outside the cut-off. We address this topic by performing 1D full wave simulations in a turbulent model plasma, comparing the model density fluctuations with those of the model reflectometry phase. In order to check whether the $1 \mathrm{D}$ results are valid on a fusion machine we study in 2D the propagation of a Gaussian beam in a snapshot of the model turbulence. Density profile and beam width parameters correspond to the set-up on Tore Supra.

A linear 1D relation can be established between the reflectometry phase fluctuation level $\sigma_{\text {rms }}$ and the density fluctuation level $\left\langle\delta n>\right.$ given the Born approximation is valid ${ }^{3}$. For O-mode launched into a linear density profile with the gradient length $L$, and a superimposed homogeneous turbulence with a Gaussian correlation function, the relation is displayed in (1).

$$
\sigma_{\mathrm{rms}}=k_{0} \sqrt{L \lambda_{\mathrm{F}}} \frac{<\delta n>}{n_{\mathrm{c}}}, \quad \lambda_{\mathrm{F}}=l_{c} \sqrt{\pi}\left[\ln \left(\frac{8 L}{\pi l_{c}}\right)-\frac{\gamma}{2}+1\right]
$$

This is a refined version of the formula previously published by Gusakov et al ${ }^{4}$, where $k_{0}$ and $n_{\mathrm{c}}$ are the vacuum wavenumber and the cut-off density, respectively, for the launched microwave, $l_{c}$ is the correlation length, and $\gamma \approx 0.577$ (Euler constant). The interdependency between $\sigma_{\text {rms }}$ and $\langle\delta n\rangle$ becomes nonlinear at high density fluctuation levels due to Bragg backscattering, which is not included in equation (1). Our strategy is, therefore, to use (1) in combination with small fluctuation levels as a benchmark for the full wave code in O-mode, which allows us to do a full-scale analysis. Since the mechanism of wave scattering is not specific to O-mode, the results can be transfered to X-mode. Increasing $\langle\delta n>$ we finally investigate the validity domain of the analytical expression.

\section{Setup and results in $1 \mathrm{D}$}

We use the numerical 1D code described by Fanack ${ }^{3}$ to solve the Helmholtz equation (2) for an

$$
\left[\frac{\mathrm{d}^{2}}{\mathrm{~d} x^{2}}+k_{0}^{2} N^{2}(x)\right] E(x)=0, \quad N^{2}(x)=1-\frac{n(x)}{n_{\mathrm{c}}}, \quad n_{\mathrm{c}}=\frac{m_{\mathrm{e}} \epsilon_{0} \omega^{2}}{e^{2}}
$$

O-mode microwave in the cold plasma approximation. The density profile $n(x)$ and the angular frequency $\omega$ of the launched wave are given. We obtain the model reflectometry phase from the 
solution for the wave electric field $E(x)$ by evaluating the phase difference between the plasma edge and the cut-off (and since there is an incident and a reflected wave we multiply by 2 ).

The input data for the plasma density is split into an average and a fluctuating part, and both are generated on a discrete grid with spacing $\Delta x$, which is typically $1 / 40$ of the vacuum wavelength.

$$
n(x)=\bar{n}(x)+\delta n_{h}(x), \quad \bar{n}(x)=x L^{-1} n_{\mathrm{c}} .
$$

Here the average density profile is linear. Since we evaluate the model phase fluctuation level $\sigma_{\text {num }}$ from the sample variance, we need different samples $h$ of the fluctuation profile.

Following Gusakov et $\mathrm{al}^{4}$ we set up homogeneous turbulence with a Gaussian correlation function. Using the correlation length $l_{c}$ from equation (1) the normalized power spectrum is

$$
\delta n_{\kappa}^{2}(k)=\text { const } \cdot \exp \left(-l_{c}^{2} k^{2} / 4\right) \text {. }
$$

Like it has been done before ${ }^{5}$ we represent the turbulence as a superposition of modes, generate the phase spectrum with random numbers, and apply the Fourier transform. Note that the longest wavelength is $2 \pi(\Delta k)^{-1}=M \Delta x$, given the fluctuation profile contains $M$ grid points. Setting $\delta n_{\kappa}(0)=0$ in the discrete spectrum we avoid to introduce an additional offset. Equation (4) is applied to the amplitude of the Fourier coefficients $\delta n_{\kappa}^{2}(\Delta k), \delta n_{\kappa}^{2}(2 \Delta k), \ldots$

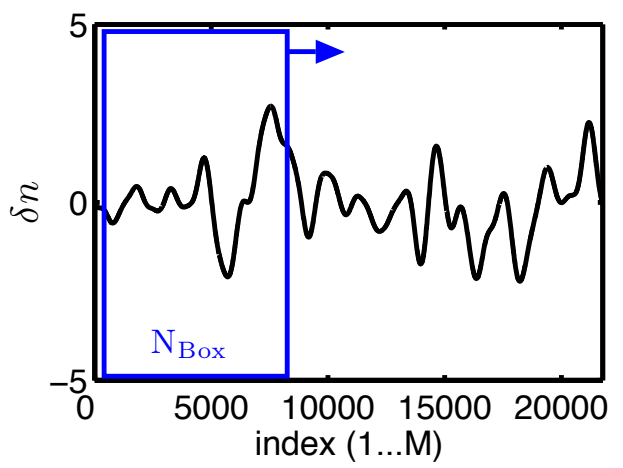

FIG. 1. Moving frame technique for a normalised fluctuation $<\delta n\rangle=1$. Subprofiles $\delta n_{h}$ are extracted using indices $h$ through $h+N_{\text {Box }}-1$. Frame count $h=1 \ldots M$. Overtraversed indices at the end are subsequentially filled from the left.

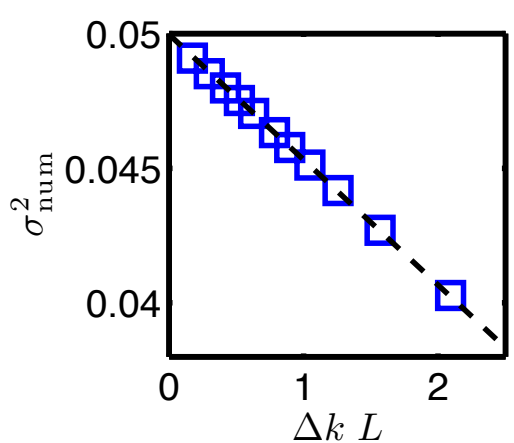

FIG. 2. Linear convergence of the square deviation $\sigma_{\text {num }}^{2}$ with $\Delta k \rightarrow 0 . L=57.7 \mathrm{~cm}, 47 \mathrm{GHz}$, $\Delta x=106 \mu \mathrm{m}, l_{c}=0.75 \mathrm{~cm},<\delta n>/ n_{\mathrm{c}}=0.001$, $N_{\text {Box }}=7000$. Squares: Scan of $M$ from 16320 to 195840. Dashed line: linear regression.
The next task is to generate different samples $h$ of the fluctuation profile $\delta n_{h}(x)$ with the given spectral properties. In our case the moving frame technique is a very compact solution: Since the number of nodes in the simulation box $N_{\text {Box }} \ll M$, it is possible to generate different instances of the fluctuation by varying the index address in the original fluctuation profile (see Fig. 1). In general this size of the simulation box is sufficient ( $\lambda_{0}$ vacuum wavelength):

$$
N_{\text {Box }} \cdot \Delta x \gtrsim L+20 \lambda_{0}
$$

Note also that the square deviation of $\delta n$ as exemplarily shown in Fig. 1 yields exactly the model density fluctuation level squared $\langle\delta n\rangle^{2}$.

Coming to the results: We find that the square deviation $\sigma_{\text {num }}^{2}$ of the model reflectometry phase in the set of fluctuation profiles $\delta n_{h}(x)$ $(h=1,2 \ldots M)$ converges linearly (see Fig. 2 ) if $\Delta k \sim M^{-1}$ approaches zero. The model turbulent modes that subsequently come into play have wavelengths much longer than the size of the simulation box. These kinds of input data are able to induce forward scattering. In parameter scans we observe that only the product $\Delta k \cdot L$ is important. If $\Delta k \cdot L<0.1$ then the accuracy of the numerical result is better than $1 \%$. 
For this estimation we regarded the value of $\sigma_{\text {num }}^{2}$ extrapolated towards $\Delta k=0$ as the nominal value (see Fig. 2). Note that the numerical computation becomes more expensive for large $L$, since the amount of turbulence data grows with $M \cdot N_{\text {Box }}$.

Finally we compare the simulation result $\sigma_{\text {num }}$ to the theoretical value $\sigma_{\text {rms }}$ from equation (1) scanning all free parameters. In order to save computation time we use $\Delta k \cdot L \approx 0.9$, which corresponds to $10 \%$ accuracy. The result in Fig. 3 shows that the dependencies on $k_{0}, L, l_{c}$, and $<\delta n>/ n_{\mathrm{c}}$ are in good agreement. For relative fluctuation levels $<\delta n>/ n_{\mathrm{c}} \gtrsim 4 \%$ the numerical result starts to deviate systematically. If it is greater than $10 \%$ then the deviation is also $>10 \%$.

FIG. 3. Phase fluctuation $\sigma_{\text {num }}$ from the simulation and $\sigma_{\text {rms }}$ from equation (1) compared. The cross-hair marks the starting point for the parameter scans: $L=57.7 \mathrm{~cm}, 47 \mathrm{GHz}, l_{c}=3 \mathrm{~cm},<\delta n>$ $/ n_{\mathrm{c}}=0.001$. X symbols: scan of $\left\langle\delta n>/ n_{\mathrm{c}}\right.$ from $5 \cdot 10^{-4}$ until 0.1 . Open circles: frequencies 24 and $58 \mathrm{GHz}$. + signs: $l_{c}=1 \mathrm{~cm}$ and $4.5 \mathrm{~cm}$. Blue triangles: $L=32.1 \mathrm{~cm}$ and $100 \mathrm{~cm}$. For better orientation the relation $\sigma_{\text {num }}=\sigma_{\text {rms }}$ is overplotted using a dashed line.

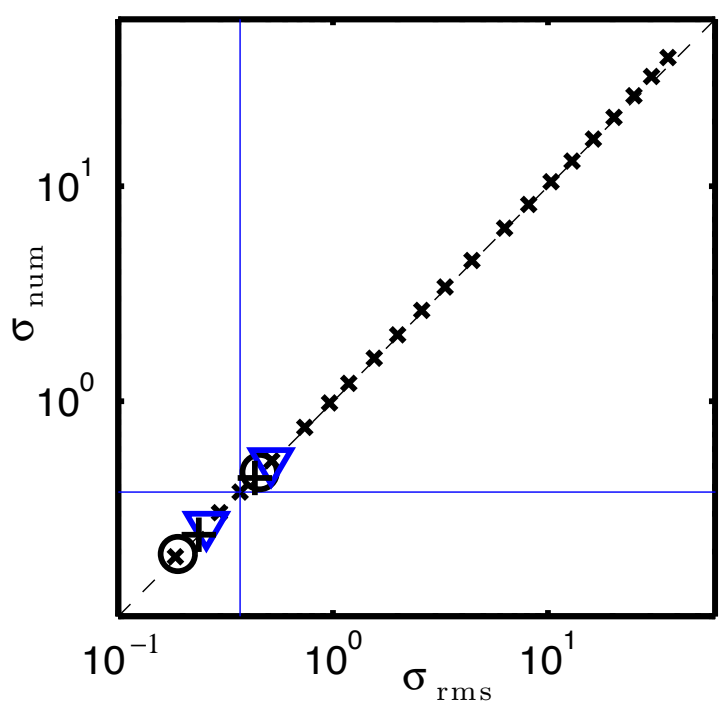

\section{Numerical 2D study on Gaussian beam propagation}

In the plasma center of Tore Supra ${ }^{6}$ the density fluctuation level normalised to the local average density is small enough for equation (1) to be applicable. This is not the case at the plasma edge, where the relative level is of the order $10 \%$ (compare Fig. 4). We study the broadening of the reflectometry beam (width $\approx 10 \mathrm{~cm}$ ) crossing the strongly fluctuating edge in order to probe the center. In addition we verify the coherency of the phase front in the direction perpendicular to the direction of propagation. Our tool is the 2D finite-differences timedomain code described by da Silva et al. ${ }^{7}$

We set up an isotropic turbulence spectrum as proposed by Hennequin et al: ${ }^{8}$

$$
\delta n_{\kappa}^{2}(k) \sim \exp \left[-4\left(k_{x}^{2}+k_{y}^{2}\right)^{0.5} \rho_{i}\right]
$$

where $\rho_{i}=0.75 \mathrm{~mm}$ is the ion Larmor radius. For $|k|<4 \mathrm{~cm}^{-1}$ we assume the spectrum to be constant. The density fluctuation is generated similarly to the $1 \mathrm{D}$ case, but for simplicity we use only a single frozen pattern of the turbulence. The envelope function (Fig. 4) is multiplied with the fluctuation in order to generate the inhomogeneity. Since the envelope is broad compared to the correlation length, we neglect that this slightly modifies

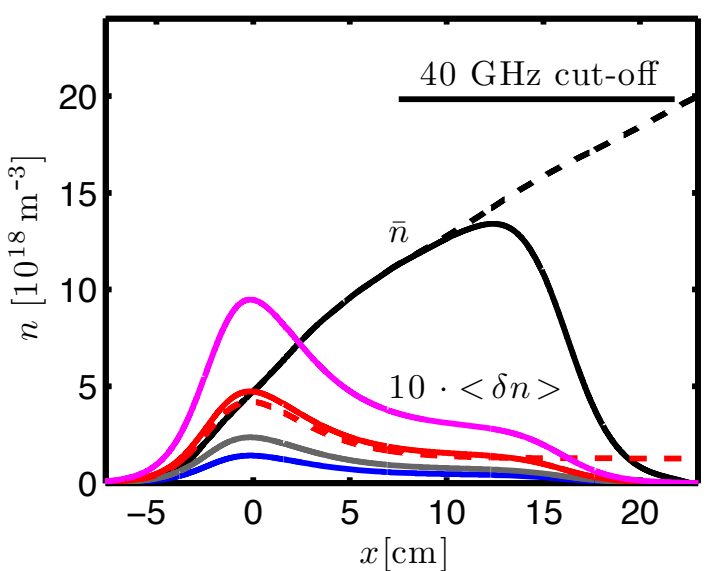

FIG. 4. Radial profiles of the average density and fluctuation envelope. ${ }^{6}$ Both are attenuated artificially at $x=16 \mathrm{~cm}$, before the cut-off, damping length $\approx 7 \lambda_{0}$. The fluctuation envelopes are magnified by 10 for better visibility. They correspond to relative fluctuation levels at the edge $(x=0)$ of $3,5,10$, and $20 \%$. Dashed lines indicate the original measurements. ${ }^{6}$ In $y$ direction (perpendicular to this sheet) these profiles are constant. 


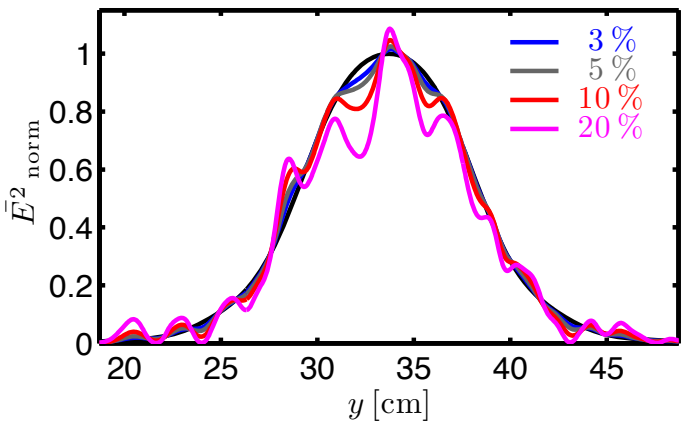

FIG. 5. Beam profiles in the direction $y$ perpendicular to the direction of propagation. Displayed is the electric field amplitude squared, averaged in time, at $x \approx 26 \mathrm{~cm}$ (see Fig. 4). Solid black line: Reference beam in the absence of fluctuations. Colored lines: Beam profiles corresponding to the different fluctuation levels from Fig. 4, and normalised to the reference.

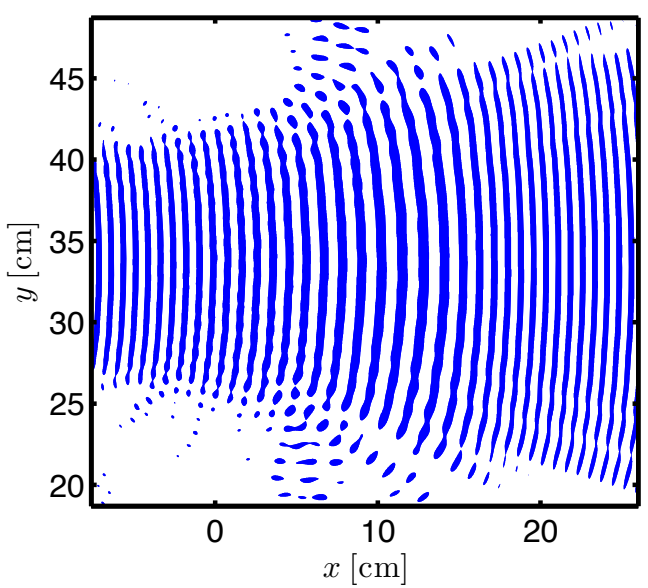

FIG. 6. Contour plot of the positive half wave of the electric field $E_{\text {norm }}>0.14$ in the $x-y$ plane. Fluctuation level 10\%.

the spectrum. Putting the model fluctuation and the average profile together we introduce an artificial attenuation shortly before the cut-off (Fig. 4) in order to mask the wave reflection.

The resulting beam profiles $(f=40 \mathrm{GHz})$ after single path transmission are displayed in Fig. 5. We find the Gaussian shape to be robust up to an edge fluctuation level of $\sim 3 \%$. Side maxima appear if the level $>5 \%$. In all cases the phase coherence remains intact (Fig. 6).

\section{Conclusions}

Using a 1D full wave code we have studied numerically the linear relation between the plasma density fluctuation level and the phase fluctuation level of a fixed frequency fluctuation reflectometer. Good agreement between the numerical results and the analytical solution in Born approximation is found. Nonlinearity due to Bragg backscattering becomes apparent as a systematic deviation from the linear relation, if the density fluctuation level is greater than $4 \%$ of the cut-off density. In plasma turbulence modelled as superposition of fourier modes the numerical phase fluctuation level squared converges linearly with decreasing discretisation in wavenumber space $\Delta k$. We identified numerically the gradient length $L$ as the important parameter for the convergence. Coherent phase fronts were found in a 2D simulation with inhomogeneous turbulence, thus confirming the applicability of the 1D result on Tore Supra. For future projects it is desirable but also demanding to verify the phase fluctuations detected by a finite antenna.

\section{Acknowledgements}

Financial support of RFBR grants 06-02-17212, 07-02-92162-CNRS, NWO-RFBR grant 047.016.015 and INTAS grant 05-8046 is acknowledged.

1 R. Nazikian, G. Kramer, and E. Valeo, Phys. Plasmas 8(5), 1840 (2001).

2 G. D. Conway et al., Proceedings of the 7th International Reflectometry Workshop, special issue of Nuclear Fusion 46(9), S665 (2006).

3 C. Fanack et al., Plasma Phys. Controlled Fusion 38, 1915 (1996); C. Fanack, Ph.D. thesis, Université Henri Poincaré, Nancy 1, 1997.
4 E. Z. Gusakov, and A. Y. Popov, Plasma Phys. Controlled Fusion 44, 2327 (2002).

5 G. Leclert et al., Plasma Phys. Controlled Fusion 48, 1389 (2006).

6 T. Gerbaud, Rev. Sci. Instrum. 77, 10E928 (2006).

7 F. da Silva et al., Nuclear Fusion 46, S816 (2006).

8 P. Hennequin et al., Plasma Phys. Controlled Fusion 46, B121 (2004). 